\title{
Prévalence De La Pression Artérielle Élevée Et Les Facteurs Associés Dans Les Départements Du Borgou Et De l’Alibori Au Bénin En 2014
}

\author{
Ahoui Séraphin \\ Faculté de Médecine (FM) de l’Université de Parakou ; Service de \\ néphrologie,Centre Hospitalier Universitaire et Départemental du \\ Borgou Parakou Bénin. \\ Vigan Jacques \\ Agboton Bruno Leopold
}

Faculté des Sciences de Santé de l'Université d'Abomey Calavi ; Clinique Universtaire de Néphrologie et d'HémodialyseCentre National Hospitalier Universitaire Cotonou Bénin.

Oké Precieux

Faculté de Médecine Université de Parakou

Codjo Léopold Houétondji

Dovonou Comlan Albert

Alassani Adébayo

Service de Médecine interne,

Centre Hospitalier Universitaire Départemental Borgou, Parakou (Bénin).

Fourn Léonard

Faculté des Sciences de Santé Université Abomey Calavi

doi: 10.19044/esj.2016.v12n33p235 URL:http://dx.doi.org/10.19044/esj.2016.v12n33p235

\begin{abstract}
Background:High blood pressure (HBP) is one of the risk factors in the occurrence of cardiovascular disease (CVD). The objective of this study was to investigate the prevalence of high blood pressure and its risk factors in the Borgou and Alibori Departments in Benin.

Methods: This was a descriptive cross-sectional study and analytical held for three months (July 1st to September 30th, 2014) and identified volunteers at least 15 years. The factors were investigated in subjects using a questionnaire providing clinical and behavioral data. The data were analyzed in Epi-info and Khi2 test was used to compare proportions with a statistically significant difference for $p<0.05$.
\end{abstract}

Results: A total of 1270 subjects were enrolled in the two departments. The mean age of subjects was $42.55 \pm 14.6$ years. The sex ratio was 0.95 . The 
prevalence of subjects with HBP was 31.10\%. The high blood pressure was significantly associated with a history of, diabetes $(p=0.005)$, with lower limb edema $(p=0.008)$, the body overload $(p=0.0000)$, smoking $(p=$ $0.002)$, the overuse and misuse of taking infusion $(\mathrm{p}=0.03)$, proteinuria $(\mathrm{p}=$ $0.03)$ and haematuria $(\mathrm{p}=0.01)$.

Conclusion:The prevalence ofhigh blood pressure is higher in Borgou and Alibori Departments in Benin and the risk factors are important. Better prevention of risk factors contribute to reduce morbidity and mortality due to cardiovascular disease.

Keywords: high blood pressure, prevalence, risk factors

\section{Résumé}

L’hypertension artérielle (HTA) constitue l'un des facteurs de risque dans la survenue d'une maladie cardio-vasculaire (MCV). L'objectif de ce travail est d'étudier la prévalence de la pression artérielle élevée (PAE) et les facteurs de risque associés dans les départements du Borgou et de l'Alibori au Bénin.

Méthodes : Il s’agissait d'une étude transversale descriptive et analytique qui s'est déroulée pendant 3 mois ( $1^{\mathrm{er}}$ juillet au 30 septembre 2014) et qui a recensé des volontaires d'au moins 15 ans. Les facteurs ont été recherchés chez les sujets à l'aide d'un questionnaire fournissant les données cliniques et comportementales. Les données ont été analysées dans Epi-info et le test de chi2 a été utilisé pour comparer les proportions avec une différence statistiquement significative pour $\mathrm{p}<0,05$.

Résultats : Au total, 1270 sujets ont été recrutés dans les deux départements. L’âge moyen des sujets était de 42,55£14,6 ans. La sex-ratio était de 0,95. La prévalence des sujets ayant une PAE était de 31,10\%. La PAE était significativement associée aux antécédents du diabète $(\mathrm{p}=0,005)$, à l'œdème des membres inférieurs ( $\mathrm{p}=0,008)$, à la surcharge corporelle $(\mathrm{p}=0,0000)$, au tabagisme $(\mathrm{p}=0,002)$, à la prise excessive et abusive de tisane $(\mathrm{p}=0,03)$, à la protéinurie $(p=0,03)$ et à l'hématurie $(p=0,01)$.

Conclusion : La prévalence de la PAE est élevée dans les départements du Borgou et de l'Alibori au Bénin et les facteurs de risque sont importants. Une meilleure prévention de ses facteurs de risque contribuera à réduire la morbidité et la mortalité dues aux maladies cardiovasculaires.

Mots clés : Pression artérielle élevée, prévalence, facteurs de risque

\section{Introduction}

L'hypertension artérielle (HTA) touche environ 20\% de la population mondiale [Murray et al. 1996]. Elle est inégalement répartie selon les 
continents et selon les pays. C’est ainsi que 15\% de la population française, $20 \%$ de la population américaine, $18 \%$ de la population chinoise sont concernées par l'HTA [Murray et al. 1996]. Dans les pays d’Afrique, 20 millions de personnes seraient affectées [WHO, 2000]. Sa prévalence estimée varie également selon les pays : 15\% pour l’Algérie, 30\% pour les îles Maurice et Seychelles, 20-35\% pour le Gabon, 9,5\% pour la Gambie [WHO, 1999]. Au Bénin, la prévalence de L’HTA était de 20,2\% en 2001. [Ministère de la Santé-PNLMNT Bénin, 2008] Elle a connu une hausse pour atteindre 27,5\% en 2008 avec les résultats de l'enquête SPEPS [OMS, 2003]. En effet, l' HTA tue, chaque année, 36 millions de personnes et elle est responsable de $63 \%$ des cas de décès à l'échelle mondiale, dont $80 \%$ surviennent dans les pays en voie de développement (PED) ou à faible revenu [Murray et al. 1996]. En plus des conséquences pathologiques, l'HTA est un facteur de risque majeur de nombreuses maladies telles que l'insuffisance cardiaque, les affections coronariennes, les accidents vasculocerebraux, l'insuffisance rénale et les rétinopathies hypertensives. Ce risque augmente quand il existe conjointement d'autres facteurs de risque tels que le diabète, l’obésité, les dyslipidémies.

Ce travail a été initié, dans les départements du Borgou et de l'Alibori, afin d'étudier la prévalence de la PAE et les facteurs associés au sein de la population en 2014.

\section{Cadre et méthode d'étude}

Notre étude s’est déroulée dans les départements du Borgou et de l'Alibori dans la partie septentrionale du Bénin. Les départements du Borgou et de l'Alibori comptent respectivement huit et six communes. Il s'agissait d'une étude transversale, descriptive et analytique en population, déroulée sur une période de trois mois, allant du $1^{\mathrm{er}}$ Juillet au 30Septembre 2014. Elle a concerné les populations des départements du Borgou et de l'Alibori qui résidaient depuis au moins six mois dans la localité. Ces populations étaient choisies sur quatre (4) sites différents dont deux (2) dans le département du Borgou et deux (2) dans le département de l'Alibori.

Etaient inclus dans l'étude, tous les habitants des départements ayant au moins15 ans d’âge retenus sur la base d'un volontariat et ayant donné leur consentement éclairé.

N’étaient pas inclus toutes les personnes incapables de répondre aux questions posées ou les sujets suivis pour une maladie rénale chronique et ceux qui avaient refusé de participer.

L’échantillonnage était exhaustif en prenant tous les participants répondant aux critères d'inclusion. Pourtant une taille minimale de 338 personnes a été retenue par site grâce à la formule de Schwartz, à partir 
d’une prévalence de 27,5\% de la PAE rapportée par l'enquête SPEPS a servi de base de référence [OMS,2003 ]

Le mode du recrutement des sujets a été basé sur le volontariat dans les deux communes de chaque département. Les participants ont été informés à l'avance des lieux et des jours du dépistage. Ce mode a été déjà utilisé par d'autres auteurs pour les dépistages de masse des facteurs de risque de la PAE [Sumaili 2009 ; Wei C et al. 2009].

Le choix des communes était aléatoire à raison de deux communes par département. Les communes de Malanville et de Karimama étaient retenues dans l'Alibori et celles de Tchaourou et de N'dali dans le Borgou. Pour chaque commune retenue l'enquête a eu lieu à la place publique de l'arrondissement central. Les activités de dépistage démarraient à partir de sept heures et prenaient fin quand il n’y avait plus de participants. La durée moyenne était de six heures.

La variable dépendante :La pression artérielle élevée (PAE) est la variable dépendante principale. Tous les sujets qui ont une pression artérielle systolique (PAS) $\geq 140 \mathrm{mmHg}$ et/ou une pression artérielle diastolique $(\mathrm{PAD}) \geq 90 \mathrm{mmHg}$ le jour de l'enquête sont considérés comme ayant une PAE [Foucarde et al . 2007]. La variable dépendante secondaire est l'HTA connue. Est considéré comme hypertendu tout sujet aux antécédents d’HTA (confirmé par un agent de santé) sous traitement antihypertenseur ou non. Il s'agit d'une variable binaire codée oui ou non. . Il s’agit d'une variable binaire codée oui ou non.

Les variables indépendantes étaient: les facteurs sociodémographiques (âge, sexe), les antécédents médicaux (diabète sucré, œdème des membres inférieurs), les variables cliniques ( la prise du poids et de la taille avec calcul de l'IMC et étude du sédiment urinaire) et les informations relatives aux habitudes comportementales (alcool, tabac, tisanes) ont été recensées.

La pression artérielle a été mesurée chez le sujet assis après 15 minutes de repos à l'aide d'un tensiomètre numérique automatique muni d'un brassard (appartenant à la liste des tensiomètres validés par la Société Française de l'Hypertension artérielle). Elle a été prise au bras droit nu, placé sur une table, paume tournée vers le haut Elle a été prise au bras droit nu, placé sur une table, et paume tournée vers le haut. Trois mesures ont été prises à des intervalles de 5 minutes ; la moyenne des deux dernières mesures est la pression artérielle retenue pour chaque participant. La pression artérielle est considérée comme élevée lorsqu’elle est supérieure ou égale à 140/90mmHg.

L’IMC a été obtenu par le quotient Poids (en Kg)/Taille au carré $\left(\mathrm{m}^{2}\right)$. Le seuil d’IMC supérieur ou égal $30 \mathrm{~kg} / \mathrm{m}^{2}$ est défini comme l’obésité tandis que le surpoids est retenu si ce seuil est compris entre 25 et $30 \mathrm{~kg} / \mathrm{m}^{2}$, 
la normale est comprise entre $18,5 \mathrm{~kg} / \mathrm{m}^{2}$ et $24,9 \mathrm{~kg} / \mathrm{m}^{2}$ et la maigreur est définie par une valeur inférieure $18,5 \mathrm{~kg}$.

La recherche de la protéinurie ou de l’hématurie ou la leucocyturie ou de la nitriturie ou de la glycosurie a consisté à immerger la bandelette réactive brièvement dans l'urine. Egoutter la bandelette en passant la tranche contre le bord du récipient. Après 60 à 90 secondes, on a procédé à la lecture de la couleur de la zone réactive avec la gamme colorimétrique de l'étiquette. Les modalités de résultats sont "positive" ou "négative". (Positive au moins une + en présence de protéinurie ou d'hématurie ou leucocyturie et négative en absence d'elles).

Un questionnaire portant toutes les variables décrites servait d'outil de collecte des données. L’équipe de collecte était constituée de 24 enquêteurs composés de huit médecins en fin de formation, 12 infirmiers, et quatre auxiliaires de santé. Avant la collecte de données proprement dite, les enquêteurs ont été préalablement formés à l'administration du questionnaire et à l'usage des différents matériels par un médecin néphrologue.

La prise des mesures physiques, le test à la bandelette urinaire et le remplissage des fiches de collecte ont été réalisés par les infirmiers préalablement formés. Les médecins avaient interprétés les résultats.

Les données collectées ont été saisies grâce au logiciel Epi data 3.1fr. L'analyse des données a été faite en utilisant le logiciel Epi info version 7. Les moyennes sont présentées avec leur écart-type et les proportions/fréquences avec leur intervalle de confiance. Le test statistique de Chi-Deux de Pearson (quand les effectifs théoriques >5) a été utilisé, pour comparer les prévalences selon les modalités. Les moyennes ont été comparées par le test $t$ de Student. La différence est statistiquement significative pour une valeur de $p$ inférieure à 0,05.

\section{Résultats}

\section{Prévalence de la pression artérielle élevée}

Sur 1270 sujets inclus dans l'étude, 395 soit 31,10\% avaient une pression artérielle élevée et 241 sujets soit 19,01\% étaient des hypertendus connus et sous traitement.

\section{Caractéristiques générales des sujets enquêtés \\ Tous les résultats sont résumés dans le tableau $\mathrm{N}^{\circ} \mathrm{I}$ Description de la population d'étude}

Dans notre série 1270 participants étaient enquêtés. Parmi eux 619 (48,74\%) étaient de sexe masculin et 651 (51,26\%) de sexe féminin avec une sex-ratio de 0,95 . Ces sujets étaient âgés de 15 à 72 ans avec une moyenne

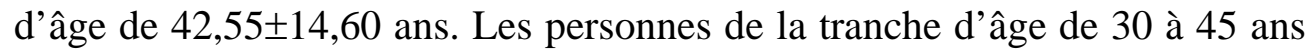
étaient majoritairement représentées (54,72\%). 
Caractéristiques relatives aux antécédents de maladie des enquêtés

Les participants qui se connaissaient hypertendus ou diabétiques étaient retrouvés respectivement dans $19,01 \%$ et 5,02\% des cas. Les antécédents d'hématurie ou des œdèmes des membres inférieurs étaient reconnus respectivement par 2,21 et 2,93\% des participants.

\section{Caractéristiques relatives au mode de vie des enquêtés}

Dans notre étude, 20,01\% des participants consommaient du tabac, $30,36 \%$ de l'alcool, 33,62\% faisaient de l'automédication et $60,31 \%$ se traitaient régulièrement à la tisane. Il ressort de même que 39,29\% ne pratiquaient pas une activité physique régulière et $51,73 \%$ ne consommaient pas régulièrement du fruit.

La consommation excessivement du repas salé, sucré et gras était respectivement de $33,62 \%$, 28,82\% et $69,61 \%$.

\section{Caractéristiques cliniques des sujets enquêtés}

Parmi les sujets de notre étude $8,06 \%$ présentaient des œdèmes des membres inférieurs (OMI). Par rapport à l'indice de masse corporelle (IMC), 614 étaient en surpoids soit 48,35\% et 151 obèses soit 11,89\%. Donc une surcharge pondérale était retrouvée chez 765 participants soit 60,24\%.

La protéinurie, l'hématurie, la leucocyturie, la nitriturie et la glycosurie étaient respectivement retrouvée chez 14,32\%, 10,86\%, 5,35\%, $2,02 \%$ et $3,74 \%$ des sujets.

\section{Relation entre la pression artérielle élevée et les facteurs de risque}

La PAE était associée aux antécédents d'HTA dans 86,02 \% $(\mathrm{p}=0,0000)$. Elle était associée aux antécédents de diabète, aux œdèmes des membres inférieurs (OMI) respectivement dans 45,24\% ( $\mathrm{p}=0,05), 31,74 \%$ $(\mathrm{p}=0,008)$ des cas. La PAE était associée à la surcharge pondérale dans $46,03 \%(\mathrm{p}=0,0000)$ des cas. Dans notre série, la PAE était aussi associée à la prise excessive et abusive de tisane dans $11,22 \%$ des cas $(\mathrm{p}=0,03)$ et au tabagisme soit $64,17 \%$ des cas $(\mathrm{p}=0,002)$. Par rapport au sédiment urinaire, la PAE était liée à la protéinurie et à l'hématurie respectivement dans $18,75 \%(p=0,003)$ et $22,97 \%(p=0,01)$ des cas. Le tableau $N^{\circ} I I$ traduit les relations entre l'HTA et les facteurs de risque..

\section{Discussions}

\section{Caractéristiques de l'échantillon}

L’âge moyen des enquêtés était de $42,5 \pm 14,6$ ans. . Il s'agissait donc d'une population relativement jeune. Elle constitue la couche la plus active, pourvoyeuse de ressources nécessaires au développement économique des deux départements. Cette moyenne d’âge présente un risque de développer 
une PAE qui serait responsable d'une insuffisance rénale chronique comme l’a constaté Sumaili en RDC [Sumaili, 2009].

\section{Caractéristiques cliniques}

La prévalence de la PAE dans notre étude corrobore celle de Ahoui [Ahoui S, 2011] qui avait retrouvé une prévalence de 35,48 \% dans la population sénégalaise. Dans notre série, la PAE était associée aux antécédents de diabète $(p=0,05)$, aux œdèmes des membres inférieurs $(\mathrm{p}=0,008)$, à la surcharge pondérale $(\mathrm{p}=0,0000)$ à la protéinurie $(\mathrm{p}=0,03)$ protéinurie et à l'hématurie $(\mathrm{p}=0,01)$. L'effet délétère de la pression artérielle élevée, sur la fonction rénale est bien documenté depuis des décennies [Fourcade et al., 2007]. La PAE quelle que soit son étiologie constitue un facteur de risque vasculaire et représente une cause importante de l’insuffisance rénale [Martins et al., 2012].

Parmi les participants soit $5,02 \%$ étaient diabétiques $(p=0,05)$,. La néphropathie diabétique constitue la principale complication rénale du diabète avec la présence d'une hypertension artérielle. Elle atteint 35\% des diabétiques et évolue vers une insuffisance rénale chronique (IRC). Elle multiplie par dix la morbidité et la mortalité cardio-vasculaires [Levey et al., 2007, Coresh et al., 2003] .

L’étude a révélé $8,06 \%$ des sujets qui avaient présenté des œdèmes des membres inférieurs $(p=0,008)$,. Les participants avec des œdèmes pourraient avoir des néphropathies glomérulaires ou en faveur des glomérulonéphrites chroniques ou autres affections avec une retention hydro-sodée.

La protéinurie était retrouvée chez 14,32\%. Sumaili et al. en RDC, dans la population de Kinshasa, avaient retrouvé une proportion de 17,1\% [Sumaili et al.,2009] et Wei C et al. avaient eu 16.2\% dans la population tibétaine [Wei Cet al.2009 Ce qui est en faveur des glomerulonephrites. La protéinurie demeure nocive pour le rein.

\section{Caractéristiques comportementales}

Dans notre étude, 20\% des participants consommaient le tabac (prisé ou chiqué). Ce résultat est en adéquation avec ceux trouvés par Bah et al., en 2006 et Sumaili en 2009 [Bah et al. 2006, Sumaili, 2009]. Le tabac a une influence sur l'hémodynamique intra rénale en augmentant le tonus sympathique et la résistance vasculaire rénale [Chase et al., 1991]. Le tabac connu comme facteur de risque cardiovasculaire est à lui seul un facteur de risque de dégradation de la fonction rénale [Hallan, 2011].Il multiplierait par 4 le risque d'atteindre le stade 5 de l'insuffisance rénale chronique.

Dans notre série, les sujets buvant de l'alcool étaient de 30,36\%. En effet selon certains auteurs la consommation modérée d’alcool protègerait 
l'être humain des maladies cardiovasculaires[Schaeffer, 2005]. Cependant la consommation excessive d'alcool pourrait entrainer des effets délétères sur les fonctions rénales [Schaeffer, 2005].

La prise abusive et régulière de la tisane était retrouvée chez 60,31\% des participants. Notre résultat est similaire à celui trouvé, au Congo Démocratique par Sumaili [Sumaili, 2009]. En effet les plantes et racines composant les tisanes étant mal connue, certaines d'entre elles pourraient contenir des métaux lourds ou des substances dangereuses et vasoconstrictrices capables d'induire et d'entretenir une PAE.

\section{Conclusion}

Cette étude montre que la prévalence de la PAE était élévée dans le Borgou et l'Alibori . Il se dégage que la prévention demeure le seul moyen de lutte efficace et devrait être axée sur la sensibilisation, le dépistage et le traitement des facteurs de risque.

\section{References :}

1. Ahoui S. Prévalence des facteurs de risque de la maladie rénale chronique au Sénégal. Mémoire de fin de CES en Néphrologie, Université Cheikh Anta Diop de Dakar2011, 64-97.

2. Bah AO, Kaba ML, Diallo MB, Kake A, Balade ML, Keita K, et al. Morbidité et mortalité hospitalières dans le service de néphrologie de l'hôpital national Donka. Mali médical 2006 ; 21(14) : 42

3. Chase HP, Garg SK, Marshall G, Berg CL Cigarette smoking increases the risk of albuminuria among subjects with type I diabete. JAMA 1991; 257: 614-7

4. Fourcade LP, Mafart PB. Hypertension aetérielle en Afrique Subsaharienne. Med Trop 2007 ;167: 559-67

5. Hallan SI Tabac et insuffisance rénale : quelles risques et quelles solutions. Kidney Int 2011; 57 :128-35

6. Levey AS, Atkins R, Coresh JChronic kidney disease as a global public health problem: approaches and initiatives - a position statement from Kidney Disease Improving Global Outcomes. Kidney Int 2007; 72:247- 259.

7. Martins DMD, Agodoa LMS, Norris KLM. Maladie rénale chronique hypertensive chez les Afro-américains: stratégies d'amélioration des soins. Cleve Clin J med 2012; 79 (10) : 725-34

8. Ministère de la Santé, Direction Nationale de la Protection Sanitaire, Programme National de Lutte contre les Maladies Non Transmissibles [MS/PNLMNT Bénin]. Rapport final de l'enquête STEPS au Bénin. Cotonou :(2008). http://www.who.int/chp/steps/2008_STEPS_Report_Benin.pdf 
consulté le 15 Janvier 2016) Murray CJL, Alan D. The Global Burden of Disease: A Comprehensive Assessment of Mortality and Disability from Diseases, Injuries, and Risk Factors in I990 and Projected to 2020, World Health Organization, Harvard School of Health, World Bank, Geneva,(1996), 1(1): 2327

9. Organisation mondiale de la Santé(OMS). Stratégie de coopération de l’OMS avec les Pays : Bénin : 2004- 2008 Cotonou (2003): 11. http://www.who.int/country-cooperation/what-who-does/strategiesand-briefs/en/ ( consulté le 15 Janvier 2016)

10. Schaeffer E Archives of alcoohol. Med Int 2005; 165: 1048-53

11. Sumaili EK. Epidémiologie de la maladie rénale chronique à Kinshasa (RDC). Thèse : médecine Université de Liège ; 2009 :39

12. Sumaili EK, Cohen EP, Zinga CV, et al.High prevalence of undiagnosed chronic kidney disease in sub- Saharan Africa: Results from Kinshasa. Nephrol Dial Transplant 2009; 24:117-123.

13. Wei C , Qinghua L, Hui W, Weiqing C, Richard JJ,Xiuqing D et al. Prevalence and risk factors of chronic kidney disease: a population study in the Tibetan population Nephrol. Dial. Transplant. (2011) 26 (5): 1592-1599 first published online October 12, 2010 doi:10.1093/ndt/gfq608

14. WHO, Report of the consultative meeting on hypertension control in the African region, World Health Organization, Regional Office for Africa, Harare (1999) www.afro.who.int/index.php?option=com..(consulté le 15 Janvier 2016).

15. WHO, Noncommunicable diseases: a strategy for the African Region, , World Health Organization, Regional Office for Africa, Harare (2000) (AFR/RC50/10) www.who.int/.../africa_ncds_background_paper.pdf (consulté le 15 Janvier 2016)

Tableau $N^{\circ}$ I Caractéristiques générales des sujets enquêtés

\begin{tabular}{ccc}
\hline Variables & $\begin{array}{c}\text { Effectifs } \\
(\mathrm{n}=1270)\end{array}$ & Fréquences (\%) \\
Sexe & & \\
Féminin & 651 & 51,26 \\
Masculin & 619 & 48,74 \\
Âge $(\mathrm{an})$ & & \\
{$[15-30[$} & 120 & 9,43 \\
{$[30-45[$} & 695 & 54,72 \\
{$[45-60[$} & 431 & 33,96 \\
$\geq 60$ & 24 & 1,89 \\
& Antécédents de maladie & \\
Hypertension artérielle & 241 & 19,01
\end{tabular}


Diabète

64

Edèmes des membres inférieurs

Hématurie

Prise régulière de tisane

Mode de vie

Consommation du tabac

Consommation de l'alcool

Automédication

386

30,36

Inactivité physique

427

33,62

39,29

Consommation irrégulière du fruit

499

51,73

Consommation excessive du repas salé

657

33,62

Consommation excessive du repas sucré

426

28,82

Consommation excessive du repas gras

366

884

69,61

Caractéristiques cliniques

Edèmes des membres inférieurs

102

8,06

Indice de masse corporelle

$\begin{array}{ccc}\text { Surpoids } & 614 & 48,35 \\ \text { Obésité } & 151 & 11,89 \\ \text { Normal } & 531 & 38,75 \\ \text { Maigreur } & 13 & 1,01 \\ \text { la Bandelette urinaire } & & \\ \text { Protéinurie } & 182 & 14,32 \\ \text { Hématurie } & 138 & 10,86 \\ \text { Leucocyturie } & 68 & 5,35 \\ \text { Nitriturie } & 26 & 2,02 \\ \text { Glycosurie } & 47 & 3,74\end{array}$

Tableau II : Relation entre la pression artérielle élevée(PAE) et ses facteurs de risque dans les départements du Borgou et de l’Alibori au Bénin en 2014 (n=1270)

\begin{tabular}{|c|c|c|c|c|}
\hline \multicolumn{5}{|c|}{ les départements du Borgou et de l'Alibori au Bénin en 2014 ( $n=1270)$} \\
\hline \multirow[t]{2}{*}{ Variables } & \multirow{2}{*}{$\begin{array}{c}\text { Total } \\
(\mathrm{N})\end{array}$} & \multicolumn{2}{|c|}{ Pression artérielle élevée } & \multirow[t]{2}{*}{$p$-value } \\
\hline & & Effectif (n) & Fréquence (\%) & \\
\hline Antécédents de diabète & & & & 0,05 \\
\hline Oui* & 64 & 28 & 45,24 & \\
\hline Non & 1206 & 367 & 44,26 & \\
\hline Edèmes des membres inférieurs & & & & 0,008 \\
\hline Oui* & 102 & 32 & 31,74 & \\
\hline Non & 1168 & 363 & 31,08 & \\
\hline surcharge pondérale & & & & 0,0000 \\
\hline Oui* & 765 & 220 & 46,03 & \\
\hline Non & 905 & 175 & 19,34 & \\
\hline protéinurie & & & & 0,003 \\
\hline Oui* & 182 & 34 & 18,75 & \\
\hline Non & 1088 & 361 & 33,18 & \\
\hline hématurie & & & & 0,01 \\
\hline Oui* & 138 & 32 & 22,97 & \\
\hline Non & 1196 & 363 & 30,35 & \\
\hline Tabac & & & & 0,002 \\
\hline Oui* & 254 & 163 & 64,17 & \\
\hline Non & 1016 & 125 & 12,30 & \\
\hline Tisane & & & & 0,03 \\
\hline Oui* & 766 & 86 & 11,22 & \\
\hline Non & 504 & 95 & 18,85 & \\
\hline
\end{tabular}

p-value

Résultats de la Bandelette urinaire

*Modalité de référence 